\title{
Cost-Effectiveness of Sarilumab Added to Methotrexate in the Treatment of Adult Patients with Moderately to Severely Active Rheumatoid Arthritis Who Have Inadequate Response or Intolerance to Tumor Necrosis Factor Inhibitors
}

\author{
Noemi Muszbek, MSc; Clare Proudfoot, PhD; Marie Fournier, PhD; Chieh-I Chen, MPH; \\ Andreas Kuznik, PhD; Zsofia Kiss, MSc; Peter Gal, MSc; and Kaleb Michaud, PhD
}

\begin{abstract}
BACKGROUND: Despite a substantial number of treatment options in rheumatoid arthritis (RA) following tumor necrosis factor inhibitor (TNFi) inadequate response or intolerance (TNF-IR), a lack of clarity on the optimal approach remains. Sarilumab, a human monoclonal anti-interleukin-6 receptor alpha antibody, can be used as monotherapy or in combination with methotrexate or other conventional synthetic disease-modifying antirheumatic drugs (DMARDs) in TNF-IR patients.
\end{abstract}

OBJECTIVE: To conduct a cost-utility analysis from a U.S. health care system perspective for sarilumab subcutaneous $200 \mathrm{mg}+$ methotrexate versus abatacept + methotrexate or a bundle of TNFi + methotrexate for treatment of adult patients with moderately to severely active RA and TNF-IR.

METHODS: Analysis was conducted via individual patient simulation based on patient profiles from the TARGET trial (NCT01709578); a 6-month decision tree was followed by lifetime semi-Markov model with 6-month cycles. Treatment response at 6 months, informed by network meta-analysis, was based on American College of Rheumatology (ACR) 20/50/70 criteria; patients achieving $\geq A C R 20$ continued with current therapy, and other patients moved to the next line of biologic DMARD therapy or conventional synthetic DMARD palliative treatment. Direct costs included wholesale acquisition drug costs and administration and routine care costs. Routine care costs and quality-adjusted life-years (QALYs) were estimated by predicting the Health Assessment Questionnaire Disability Index score based on treatment response and were imputed from published equations.

RESULTS: Sarilumab + methotrexate dominated the TNFi bundle + methotrexate, achieving lower costs $(\$ 319,324$ vs. $\$ 356,096)$ and greater effectiveness (4.27 vs. 4.15 QALYs), and was on the cost-efficiency frontier with abatacept + methotrexate $(\$ 360,211$ and 4.29 QALYs). Abatacept + methotrexate was not cost-effective versus sarilumab + methotrexate. Scenario analyses indicated the results were robust; sarilumab + methotrexate became dominant against abatacept + methotrexate after reduced model horizon, minimum response based on ACR50 or ACR70, or time to discontinuation per treatment class. Sarilumab + methotrexate was also dominant versus the TNFi bundle; when class-specific time to treatment discontinuation was specified, sarilumab remained cost-effective with an incremental cost-effectiveness ratio of $\$ 36,894$.

CONCLUSIONS: Sarilumab + methotrexate can be considered an economically dominant (more effective, less costly) option versus a second TNFi+ methotrexate; compared with abatacept + methotrexate, it is a less costly but less effective option for patients with moderately to severely active RA who have previously failed TNFi.

J Manag Care Spec Pharm. 2019;25(11):1268-80

Copyright $\odot 2019$, Academy of Managed Care Pharmacy. All rights reserved.

\section{What is already known about this subject}

Guidelines for the treatment of rheumatoid arthritis (RA) issued by both the American College of Rheumatology and European League Against Rheumatism recommend that switching to a treatment with a different mechanism of action may be an appropriate approach for patients with RA who have inadequate response or intolerance to a tumor necrosis factor inhibitor (TNFi).

Sarilumab, an interleukin-6 receptor alpha inhibitor, represents an alternative mechanism of action, with efficacy and safety evaluated both as monotherapy and in combination with methotrexate or other conventional synthetic disease-modifying antirheumatic drugs.

\section{What this study adds}

This study demonstrates that, in the treatment of RA, sarilumab $200 \mathrm{mg}$ subcutaneous (SC) injection every 2 weeks (q2w) + methotrexate is an economically dominant option compared with cycling TNFi + methotrexate following TNFi inadequate response or intolerance (TNF-IR).

Abatacept $125 \mathrm{mg}$ SC once weekly+methotrexate is not costeffective compared with sarilumab $200 \mathrm{mg}$ SC q2w + methotrexate in the treatment of RA following TNFi-IR.

$\tau$ oint damage, stiffness, and swelling, as well as radiographic progression are the hallmark characteristics of rheumatoid arthritis (RA), and their sustained remission is the desired goal of treatment for enabling long-term improvement of physical function and health-related quality of life for patients with this chronic, progressive autoimmune condition. ${ }^{1}$ While early diagnosis with prompt treatment is a key factor for achieving remission, earlier time to remission is also a predictive factor for sustained clinical remission in treatment-naive patients. ${ }^{2}$ Equally time critical is adequate disease control in patients who are already on the treatment pathway, as reflected in treatment guidelines recommending prompt therapeutic change in patients who do not adequately reach treatment target. 3,4 


\section{Cost-Effectiveness of Sarilumab Added to Methotrexate in the Treatment of Adult Patients with Moderately to Severely Active Rheumatoid Arthritis Who Have Inadequate Response or Intolerance to Tumor Necrosis Factor Inhibitors}

Following an inadequate response to initial treatment with conventional synthetic disease-modifying antirheumatic drugs (csDMARDs; e.g., methotrexate, sulfasalazine, leflunomide, and hydroxychloroquine), U.S. treatment guidelines recommend the addition of a biologic DMARD (bDMARD) or a targeted synthetic DMARD., ${ }^{3,4}$ Biologic DMARDs comprise tumor necrosis factor-alpha inhibitors (TNFi), T cell costimulatory inhibitors, anti-B cell agents, and interleukin-6 receptor (IL-6R) inhibitors; targeted synthetic DMARDs comprise Janus kinase inhibitors. For patients who experience an inadequate response or intolerance to initial TNFi, U.S. and European guidelines state that switching to a treatment with a different mechanism of action (MOA) may be an appropriate approach. This strategy is supported by a significant body of evidence from claims analyses indicating beneficial outcomes associated with MOA switching, ${ }^{5-13}$ while other evidence from a randomized control trial (RCT) and other claims analyses point to conflicting evidence of enhanced outcomes associated with TNFi cycling. ${ }^{14-16}$ Nonetheless, cycling between TNFi treatments after failure with a previous TNFi remains commonplace, $, 15,17-23$ with a number of factors likely influencing clinical practice, including compliance with the health insurance mandate (e.g., step-edit requirement) and rheumatologist or patient preferences..$^{24,25}$

While rheumatologists have a substantial number of therapeutic options currently available for patients with RA, there is a lack of clarity on the optimal approach for patients who have inadequate response or intolerance to TNFi (TNF-IR). Therefore, further evidence to inform prescribing is warranted, and both clinical effectiveness and economic consequences of these options should be considered. ${ }^{24}$

Sarilumab (Kevzara), approved by the U.S. Food and Drug Administration, is a human monoclonal antibody directed against both the soluble and membrane-bound IL-6R alpha. The efficacy and safety of sarilumab have been evaluated both as monotherapy and in combination with methotrexate or other csDMARDs for the treatment of moderately or severely active RA in patients who have had an inadequate response or intolerance to 1 or more DMARDs. ${ }^{26-29}$ The present study evaluated the cost-effectiveness from a U.S. health care payer perspective of sarilumab subcutaneous (SC) $200 \mathrm{mg}$ in combination with csDMARDs, in accordance with its approved indication in the treatment of adult patients with moderately to severely active RA. Sarilumab + methotrexate was compared against abatacept or a bundle of TNFi treatments including adalimumab, certolizumab, etanercept, and golimumab, each combined with methotrexate.

\section{Methods}

The cost-utility analysis (CUA) was conducted from a U.S. commercial payer perspective in a target population of patients with RA having inadequate response or intolerance to at least 1 prior TNFi therapy and who were appropriate for switching to a bDMARD with a different MOA, including sarilumab. As RA influences both mortality and morbidity, health outcomes were measured in quality-adjusted life-years (QALYs).

\section{Model Structure and Patient Simulation}

A 6-month decision tree modeling the 6-month efficacy phase was followed by a lifetime semi-Markov state transition model with individual patient simulation (IPS) developed in Excel version 2013 (Microsoft, Redmond, WA).

Heterogeneity is a significant feature of the RA patient population $;^{30}$ therefore, for the estimation of expected patient health outcomes and costs, each patient was allowed to move between health states in a stochastic manner (i.e., using IPS). ${ }^{31}$ This approach also allowed the tracking of individual patient characteristics (e.g., age) and clinical outcomes (i.e., Health Assessment Questionnaire Disability Index [HAQ-DI] progression) over the lifetime horizon of the model (Appendix A, available in online article). ${ }^{24,32}$ For each patient in the model, a duplicate or "twin" was created for each comparator, ensuring that the comparisons were not influenced by factors such as baseline characteristics, other than the outcomes of the different treatment sequences.

The profiles of patients simulated in the arms of the model were based on patients enrolled in TARGET, a pivotal phase 3 randomized, double-blind, placebo-controlled trial of TNF-IR patients randomized to sarilumab SC in combination with csDMARDs. ${ }^{29}$ In TARGET, adult patients fulfilling the 2010 American College of Rheumatology (ACR) classification criteria for RA and with moderate to severe RA were included; other inclusion and exclusion criteria are reported elsewhere. ${ }^{29,33}$ In total, 546 eligible patients were randomized (1:1:1) to sarilumab SC $150 \mathrm{mg}$ or $200 \mathrm{mg}$ or placebo SC every 2 weeks (q2w) added to csDMARDs. Patient ages ranged from 19 to 88 years (mean $52.9 \pm 12.4$ ), $81.9 \%$ were female, and $71.1 \%$ were white. Duration of RA ranged from 0.6 to 54.0 years (mean 12.1 \pm 9.4 ), baseline HAQ-DI scores ranged from 0 to 3.0 (mean $1.8 \pm 0.6$ ), and $76.8 \%$ of the population had only 1 exposure to TNFi before randomization (Table 1). ${ }^{26,29}$

At the end of the initial 6-month period of the decision tree, patients were classified as the following: (a) a responder where ACR response was $\geq 20 \%$ ( $A C R \geq 20$ ), inclusive of patients with ACR20, ACR50, and ACR70 responses and either continued with the initial treatment or discontinued because of any other reason (e.g., adverse effects, patient preference), or (b) a nonresponder where ACR response was $<20 \%$ and the patient transitioned to the subsequent treatment line (rituximab intravenous [IV] + csDMARD); or moved to the state of death.

Once patients entered the semi-Markov model, at every subsequent 6-month interval, surviving patients then either remained on the given line of treatment, discontinued treatment/transitioned to the next line of treatment (rituximab IV + csDMARD at second line or final csDMARD palliative 


\section{Cost-Effectiveness of Sarilumab Added to Methotrexate in the Treatment of Adult Patients with Moderately to Severely Active Rheumatoid Arthritis Who Have Inadequate Response or Intolerance to Tumor Necrosis Factor Inhibitors}

targeted DMARDs in the TNF-IR population; golimumab was the only TNFi that had been examined in a TNF-IR population at the time of the study. ${ }^{35}$ Up to $48.4 \%$ of TNF-IR patients achieved minimum response for sarilumab versus $52.0 \%$ for abatacept and $38.4 \%$ for the TNFi bundle (Table 1). ${ }^{34}$

ACR response was then mapped to a relative change in HAQ-DI score, measuring disability through physical function in patients, based on response and HAQ-DI data from the MOBILITY Part B RCT [NCT01061736], because of its larger sample size relative to the TARGET RCT. ${ }^{26,36}$ Changes in HAQ-DI were then predicted for each patient within each of the ACR response categories (e.g., $\mathrm{ACR} \geq 70, \mathrm{ACR} \geq 50$, and $<70$; ACR $\geq 20$ and <50; and ACR <20). HAQ-DI scores were assumed to remain constant while a patient remained on a given bDMARD treatment. ${ }^{37}$ HAQ-DI scores for patients who discontinued a bDMARD returned to the baseline level; the response level was then predicted separately for the subsequent line of treatment, and the HAQ-DI score changed accordingly. ${ }^{37}$ HAQ-DI scores for patients on csDMARD palliative treatment were assumed to increase (i.e., worsen) annually by $0.045 .{ }^{38}$

Consistent with previously published cost-effectiveness models in RA, outcomes were independent of the line in which the treatment was administered. ${ }^{37,39,40}$ However, patients on csDMARD palliative treatment were assumed not to achieve any treatment response.

Treatment Duration. As a conservative approach, the same treatment discontinuation rate following real-world rate of discontinuation of TNFi was applied to all treatment classes. This discontinuation rate was estimated based on a de novo analysis of the Canadian RHUMADATA registry with parametric models fitted to the time to treatment discontinuation data by drug class (TNFi, IL-6R inhibitor [tocilizumab], and other MOA) and for the combined drug classes. The Gompertz, lognormal, and generalized gamma models had the best fit after consideration of Akaike and Bayesian information criteria. Based on visual comparison of the observed and predicted curves and probability plots, Gompertz distributions were selected as the best fits. This analysis showed significant difference in treatment discontinuation rates between TNFi therapies and IL-6R inhibitors, with patients on IL-6R inhibitors staying on treatment longer. Therefore, in the scenario analyses, separately fitted discontinuation curves were applied to the 3 treatment classes (TNFi, IL-6R inhibitor, and other MOA).

Utilities and Mortality. The estimation of QALYs was based on patient life expectancy and utility weights. Utility weights were applied to life-years accrued according to the HAQ-DI score and gender at each model cycle, calculated by an equation fitted on data from several trials of adalimumab ${ }^{41,42}$ : utility (health utility index-3) $=0.76-0.28 \times$ HAQ-DI $+0.05 \times$ female. For example, if HAQ-DI was 1.8 , then utility $=0.76-0.28 \times 1.8+0.05$ (for a female). Additional utility equations were tested in the sensitivity analyses. The estimation of mortality was based on U.S. life tables adjusted to RA according to changes in HAQ-DI, with general population mortality rates for males and females in the United States obtained from the National Vital Statistics Reports from 2015. ${ }^{43,44}$ Adverse events were not separately evaluated in the model as their effects were assumed to be included in the utility equations.

Costs. Health care costs were estimated in 2018 U.S. dollars, inclusive of drug cost, and costs of disease management, routine care, and drug administration (e.g., outpatient and nurse visits, costs for IV infusion). Drug costs were based on the 2018 wholesale acquisition cost (WAC) of each drug, applied to the dosing and treatment schedules specified in the prescribing information for the comparators and sarilumab clinical trials (Table 1); in the base case, no discounts were applied to the list costs.

For the computation of treatment costs, all bDMARDs were assumed to be administered in combination with methotrexate, based on the dose administered in the TARGET trial of sarilumab + methotrexate. ${ }^{26}$ The cost of the TNFi bundle was based on a weighted average of each TNFi in the bundle (46\% etanercept SC, 31\% adalimumab SC, $8 \%$ golimumab SC, and $15 \%$ certolizumab SC) as per its TNFi share. Costs of csDMARD palliative treatment were based on the weighted average cost of different csDMARDs based on proportions of patients using the respective csDMARDs. ${ }^{39}$ Wastage (i.e., no vial or pack sharing) of bDMARDs was considered in the base case, and no wastage (i.e., vial or pack sharing) was assumed in the scenario analyses. Wastage implies that if there is leftover after administering the correct dose to a patient, this leftover is discarded. Therefore, in the model, the full cost of the vial was applied to the computation of treatment cost under the assumption of wastage. While efficacy outcomes have implicitly included the treatment adherence seen in the trials (for the initial 6 months) and in clinical practice (after an initial 6 months), for costs, the rate of treatment adherence was assumed to be $100 \%$ for all treatments. This leads to potential overestimation of drug costs and, thus, a conservative approach was followed.

Disease management or routine care costs included the use of non-DMARD medications, outpatient care, and hospitalization by Medicare patients, ${ }^{45}$ with costs adjusted for age, disease duration, comorbidities, HAQ-DI score at baseline, current HAQ-DI score, gender, type of DMARD received, number of previous DMARDs, years of education, and ethnicity. These costs were inflated to 2017 U.S. dollars using the medical component of the Consumer Price Index. ${ }^{46}$

The base case analysis considered only direct costs; scenario analysis was conducted from a societal perspective, which estimated workdays lost per HAQ-DI level. ${ }^{47}$ 


\section{Cost-Effectiveness of Sarilumab Added to Methotrexate in the Treatment of Adult Patients with Moderately to Severely Active Rheumatoid Arthritis Who Have Inadequate Response or Intolerance to Tumor Necrosis Factor Inhibitors}

TABLE 2 Base Case Cost-Effectiveness Analysis Results

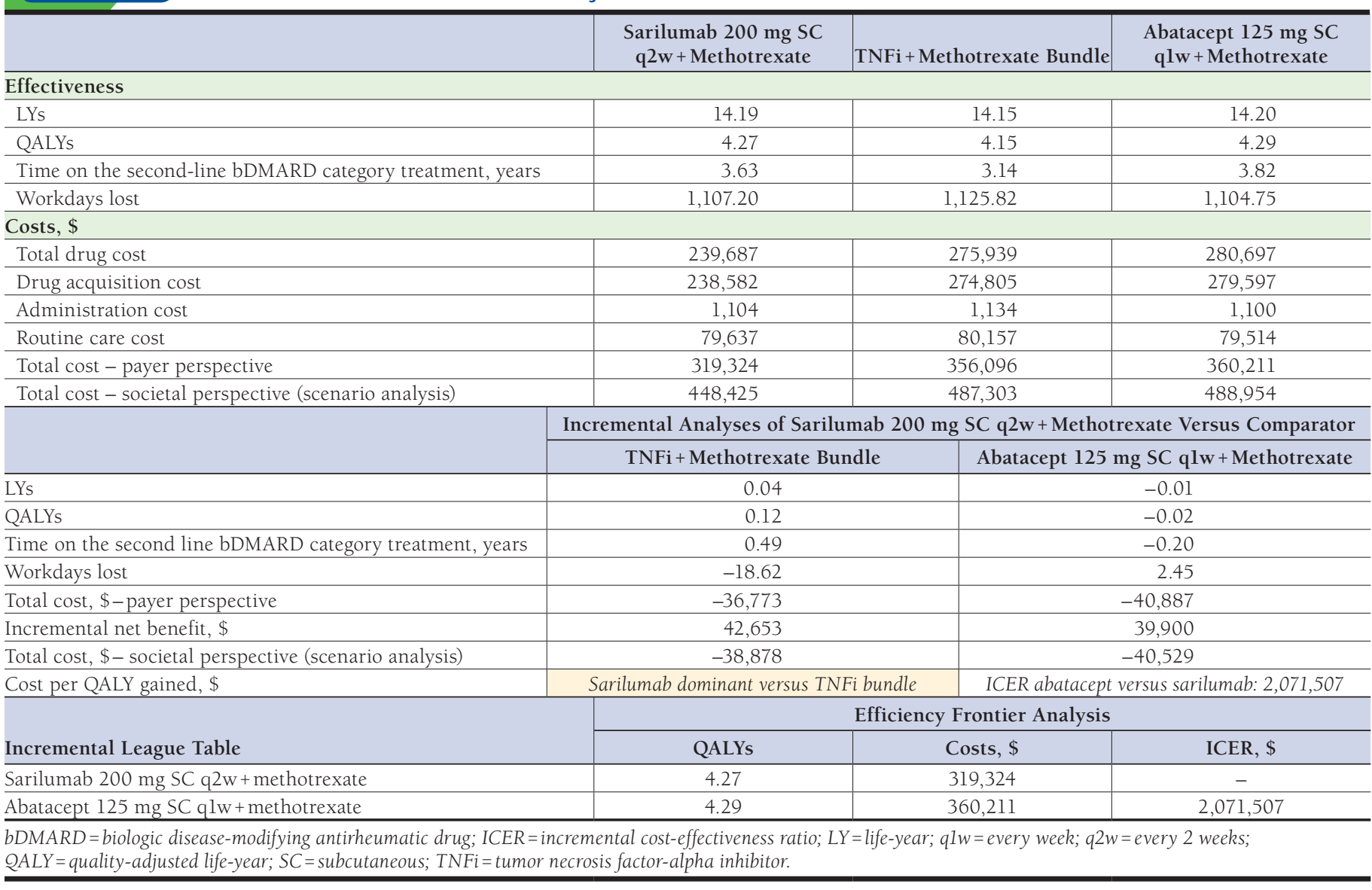

\section{Analyses}

Deterministic Analyses. Estimated health benefits (lifeyears [LYs] gained and QALYs gained) and costs were each discounted at a rate of $3 \%$ as recommended by the U.S. Panel on Cost-Effectiveness in Health and Medicine. ${ }^{48}$ Incremental cost-effectiveness ratios (ICERs) were obtained for sarilumab sequences versus any of the other comparator treatment sequences. Efficiency frontiers were computed and plotted, demonstrating treatment sequences that were not dominated (i.e., not costlier and less effective).

Scenario analyses and deterministic l-way sensitivity analyses (OWSA) were conducted on inputs and structural assumptions to account for uncertainty in model parameters. Scenario analyses evaluated the robustness of the base case results by testing key model assumptions around the time horizon, minimum response criteria, treatment discontinuation, mortality rate, and other model inputs. In addition, to represent the discounts to WACs typically negotiated for DMARDs, a further scenario was also evaluated whereby drug costs net of class-level discounts were applied to the model. The discount rates applied were based on those applied in an evidence report issued by the Institute for Clinical and Economic Review. ${ }^{24}$ In this evaluation, discounts were estimated by comparing average net prices for each agent over the 4 quarters of 2016 against its WAC. An average discount was then estimated for each drug class: $\mathrm{TNFi}=30 \%, \mathrm{CD}-20$ directed cytolytic antibody $=15 \%$, T cell inhibitors $=30 \%$, IL- 6 inhibitors $=20 \%$, Janus kinase inhibitors $=5 \% .{ }^{24}$

For the deterministic OWSA, parameters were varied to their extreme values ( \pm standard error or $95 \%$ confidence interval, or if neither was available $\pm 20 \%$ of the base case). Given the difficulty in interpreting a tornado diagram where the ICER results are in all 4 quadrants of the cost-effectiveness plane, or in the context of small QALY differences and small cost differences, incremental net benefit was estimated as incremental QALYs $\times$ threshold-incremental costs. A commonly accepted cost-effectiveness threshold of \$50,000 per QALY was specified. ${ }^{49-51}$

Probabilistic Sensitivity Analyses. Following the joint best practices guidelines from the International Society for Pharmacoeconomics and Outcomes Research and the Society 
FIGURE 1 Deterministic Sensitivity Analyses: Tornado Diagram of Incremental Net Benefit (Computed with U.S. \$50,000 Threshold) for Abatacept $125 \mathrm{mg}+$ Methotrexate Versus Sarilumab $200 \mathrm{mg}$ SC q2w+ Methotrexate

Package cost-sarilumab syringe $200 \mathrm{mg}$

Time to treatment discontinuation-RHUMADATA curves

Response rates $-\mathrm{ABT}+\mathrm{MTX} \mathrm{SC}$

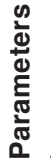

Response rates-SAR+MTX $200 \mathrm{mg}$

Mortality HR index

Annual increase of $H A Q-D I$ score while on csDMARD palliative care

Utility multiplier

HAQ-DI change on response

Routine care multiplier

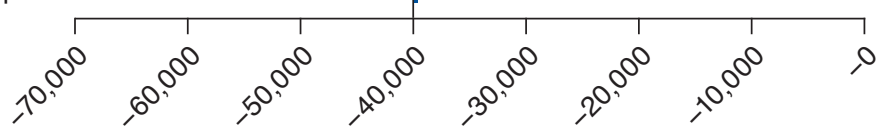

Incremental Net Benefit (U.S. \$)

$A B T=$ abatacept $;$ csDMARD = conventional synthetic disease-modifying antirheumatic drug; HAQ-DI= Health Assessment Questionnaire Disability Index; HR= hazard ratio; $M T X=$ methotrexate; $q 2 w=$ every 2 weeks; $S A R=$ sarilumab; $S C=$ subcutaneous.

for Medical Decision Making, parameter uncertainty in the model was also assessed by simultaneously varying all parameters with parameter uncertainty according to their assumed distribution (i.e., probabilistic sensitivity analyses [PSA]), based on second-order Monte Carlo simulation of 300 iterations on 500 patients. ${ }^{32,52}$ Cost-effectiveness acceptability curves were generated to depict the proportion of cost-effective simulations, or the probability of cost-effectiveness, over a range of willingness to pay thresholds.

\section{Results}

In the base case, assuming all treatment classes were ascribed equivalent time to treatment discontinuation after 6-month response, time on second line therapy was 3.63 years for sarilumab, 3.82 years for abatacept, and 3.14 years for the TNFi bundle (Table 2). Total QALYs for sarilumab were 4.27 versus 4.29 for abatacept and 4.15 for the TNFi bundle. Total costs (based on list prices) were $\$ 319,324$ for the sarilumab treatment sequence, $\$ 360,211$ for the abatacept treatment sequence, and $\$ 356,096$ for TNFi bundle treatment sequence. The sarilumab treatment sequence dominated the TNFi bundle due to achieving lower costs and higher effectiveness. Compared with the abatacept sequence, the sarilumab sequence was only slightly less effective ( -0.02 QALYs) and significantly less costly $(-\$ 40,887)$. As such, an ICER from the abatacept perspective would approach 2 million per QALY versus sarilumab (Table 2 and Appendices A and B, available in online article).

Scenario analyses indicated that the results were sensitive to the model time horizon, minimum level of ACR response, and treatment discontinuation. Sarilumab dominance versus the TNFi bundle remained under all scenarios and became dominant versus abatacept under scenarios with 24-week or 1-year time horizons, ACR50 and ACR70 responses, and treatment discontinuation according to drug class.

The scenario from a societal perspective indicated that the sarilumab sequence would result in approximately 19 fewer workdays lost versus the TNFi bundle, because of a more sustained treatment response, versus abatacept, 3 more workdays were lost for sarilumab over the course of a lifetime (Table 2). This would result in total costs of $\$ 448,425$ for the sarilumab treatment sequence, $\$ 488,954$ for the abatacept treatment sequence, and $\$ 487,303$ for TNFi bundle treatment sequence. As in the base case, the sarilumab treatment sequence would continue to dominate the TNFi bundle; however, given continued lower total costs for sarilumab, from the abatacept treatment sequence, an ICER above $\$ 2$ million would continue to result.

Finally, the discounts applied to the WAC would result in total costs of $\$ 281,939$ for the sarilumab treatment sequence, $\$ 299,549$ for the abatacept treatment sequence, and $\$ 297,479$ for TNFi bundle treatment sequence. The sarilumab treatment 

U.S. $\$ 50,000$ Threshold) for Sarilumab $200 \mathrm{mg}$ SC q2w+Methotrexate Versus TNFi Bundle

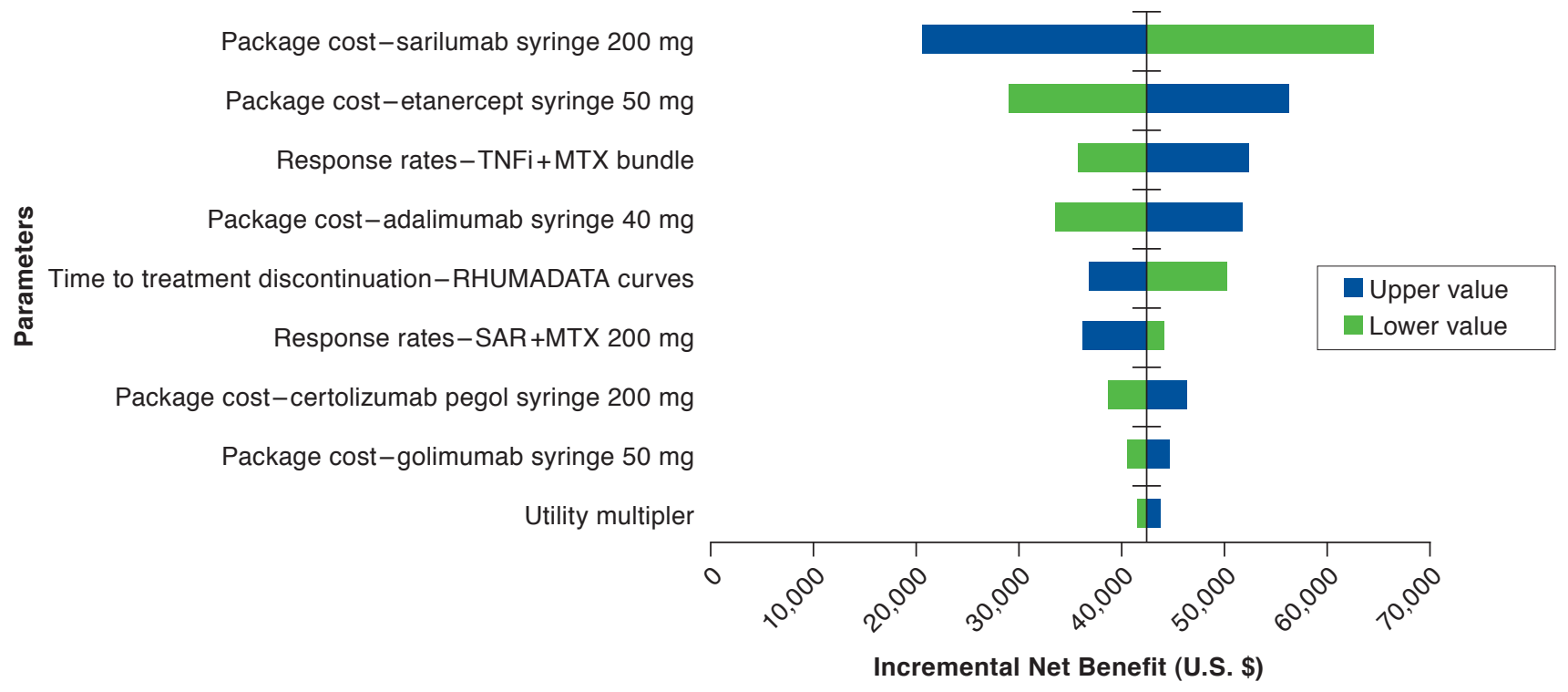

MTX=methotrexate; $q 2 w=$ every 2 weeks; SAR=sarilumab; SC=subcutaneous; TNFi=tumor necrosis factor-alpha inhibitor.

sequence would continue to dominate the TNFi bundle, while from the abatacept perspective, an ICER of $\$ 892,225$ would result versus sarilumab because of the significantly lower incremental cost of sarilumab retained in this scenario.

Deterministic OWSA revealed that results were most sensitive to sarilumab and comparators' doses (Figure 1 and Figure 2). Simulations from probabilistic sensitivity analyses for sarilumab versus abatacept suggested comparable costs and health benefits for these treatment sequences (data not presented); the cost-effectiveness acceptability curve indicated that the probability of sarilumab being cost-effective was around 70\%-80\% independently of the threshold (Figure 3).

\section{Discussion}

In a patient population with moderately or severely active RA who are TNF-IR, the present CUA indicated that a bDMARD treatment sequence initiated with sarilumab is economically dominant compared with continuation with TNFi, evaluated as class of treatments in aggregate. The treatment sequences beginning with abatacept or sarilumab in combination with methotrexate were both on the efficiency frontier, with an incremental net benefit of sarilumab versus abatacept of $\$ 39,900$ at a threshold of $\$ 50,000$ per QALY. From the perspective of abatacept versus sarilumab (where abatacept had $0.5 \%$ higher number of QALYs and 13\% higher costs), an ICER approaching \$2.1 million would result, well above ranges accepted internationally or in the United States (e.g., up to $\$ 150,000){ }^{31-33,53}$

Scenario analyses indicated that the model was robust to variations in a large number of model inputs; sarilumab continued to dominate the TNFi bundle across most scenarios, with reduced costs and greater QALYs. While dominance was not sustained for sarilumab versus the TNFi bundle when longer time to treatment discontinuation for anti-IL-6R inhibitor class of treatments was specified as per the RHUMADATA analysis results, sarilumab remained cost-effective with an ICER of $\$ 36,894$.

Versus abatacept in the scenario with increased time to discontinuation for IL-6 inhibitor class, sarilumab dominated abatacept. Sarilumab also attained dominance versus abatacept on the scenarios with shorter model horizons (e.g., 24 weeks and 1 year), because clinical benefits for abatacept only accrued later in the time horizon. Dominance also resulted when the criteria for minimum response was raised to ACR50 and ACR70.

Consistent with previous research, the results of the present analysis underscore the overall benefits of switching to an RA therapy with a different MOA rather than cycling between TNFi. ${ }^{5-13}$ A limited number of other studies have found clinical and cost benefits associated with cycling between anti-TNF drugs, possibly explained by patient response to a particular formulation versus another because of antidrug antibodies or structural and functional differences. ${ }^{14-16,54}$ The present study, 
FIGURE 3 Probabilistic Sensitivity Analyses: Cost-Effectiveness Acceptability Curves

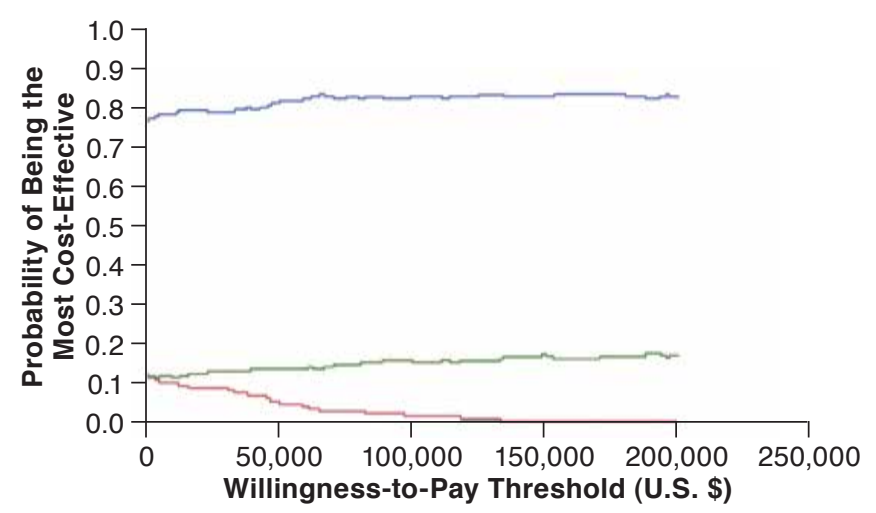

SAR+MTX $200 \mathrm{mg}-\mathrm{TNFi}+\mathrm{MTX}$ bundle $-\mathrm{ABT}+\mathrm{MTX} \mathrm{SC}$

$A B T=$ abatacept $;$ MTX = methotrexate; $S A R=$ sarilumab; $S C=$ subcutaneous; TNFi= tumor necrosis factor-alpha inhibitor.

in the short term, is based on efficacy and safety between treatments with statistically nonsignificant differences which is taken into account in the probabilistic analyses. In the long term, the model is informed by the extrapolated long-term discontinuation data influencing the time to the next treatment in the sequence. ${ }^{14-16}$

In many U.S. commercial health plans, formulary restrictions often prevail over evidence-based guidelines for actual clinical practice. ${ }^{55}$ Based on most recent data, over $60 \%$ of RA patients covered by U.S. commercial health plans are required to step through at least 2 TNFis as part of the insurer's mandated step therapy protocol before access to a non-TNFi biologic is granted, whereas clinical guidelines recommend that a non-TNFi biologic could be used immediately following any targeted DMARD failure. Comprehensive evaluations such as the present CUA continue to be warranted to inform cost-effective reimbursement decisions addressing objectives of both health care providers and payers.

Two other cost-effectiveness analyses have found sarilumab monotherapy to be cost-effective compared with comparative treatment in patients with RA but in an earlier setting (i.e., patients intolerant to or inadequate responders to csDMARDs). ${ }^{56,57}$

\section{Limitations}

Some limitations of this study must be considered. There is inherent uncertainty in the evidence regarding short-term treatment response rates and long-term benefits of bDMARDs. For example, the 6-month response rates applied to the model were based on an NMA, and there are limitations in applying
NMA results for $1 \mathrm{TNFi}$, golimumab, as a proxy for the TNFi class. This approach was taken because of the lack of comparative efficacy data for some of the TNFis in the TNF-IR population, with costs estimated on a market share-based weighted average of the agents within the bundle.

In addition, response rates estimated from the RA trials included in the NMA can be affected by a heterogeneous trial population and placebo arm response rates that have proven to be heterogeneous across trials. ${ }^{58}$ However, these issues have been considered and mitigated via metaregression analysis in the NMA that informed the present analysis.. ${ }^{53}$ A further measure to account for RA patient heterogeneity was taken by applying IPS to the real-world, heterogeneous patient population from the TARGET international RCT (which included patients from the United States), duplicated between the comparator arms, and tracking these patients across the lifetime horizon of the model. ${ }^{59}$ This approach, which added to the model's external validity, was in contrast to the homogeneous RA patient cohort that was modeled in the evidence appraisal of RA biologics published in the report issued by the Institute for Clinical and Economic Review. ${ }^{24} \mathrm{~A}$ further contrast between the present analysis and the institute's evaluation was the assumption in the present model that patients could discontinue treatment because of a variety of reasons, whereas, in the institute's evaluation, treatment discontinuation by patients was not a factor that was accounted for in the model.

Consistent with previously published cost-effectiveness models in RA, the initial 6-month treatment response was based on ACR 20/50/70 response criteria and assumed to be independent to the line of treatment. ${ }^{37}$ This conservative assumption ascribed to the model addressed the limited evidence on efficacy per line of treatment, despite other contrasting evidence suggesting that efficacy of TNFis is reduced with each subsequent TNFi. $6,7,22$

Finally, the additional strengths of the current cost-effectiveness analysis include its conduct according to best practice guidelines.$^{60}$ Furthermore, the results remained robust in various sensitivity analyses.

\section{Conclusions}

For patients with moderately or severely active RA who have previously failed TNFi treatments, sarilumab $200 \mathrm{mg}$ SC $\mathrm{q} 2 \mathrm{w}+$ methotrexate can be considered an economically dominant treatment option that is associated with lower costs and higher health benefit compared with a subsequent treatment of TNFi combined with methotrexate, while abatacept $125 \mathrm{mg} \mathrm{SC} \mathrm{qlw}+$ methotrexate is not cost-effective compared with sarilumab $200 \mathrm{mg} \mathrm{SC} \mathrm{q2w+methotrexate.}$ 


\section{Cost-Effectiveness of Sarilumab Added to Methotrexate in the Treatment of Adult Patients with Moderately to Severely Active Rheumatoid Arthritis Who Have Inadequate Response or Intolerance to Tumor Necrosis Factor Inhibitors}

\section{Authors}

NOEMI MUSZBEK, MSc, and ZSOFIA KISS, MSc, Evidera, London, United Kingdom. CLARE PROUDFOOT, PhD, Sanofi, Guildford, United Kingdom; and MARIE FOURNIER, PhD, Sanofi, Chilly-Mazarin, France. CHIEH-I CHEN, MPH, and ANDREAS KUZNIK, PhD, Regeneron Pharmaceuticals, Tarrytown, New York. PETER GAL, MSc, Evidera, Budapest, Hungary; KALEB MICHAUD, PhD, University of Nebraska Medical Center, Omaha, and FORWARD, The National Databank for Rheumatic Diseases, Wichita, Kansas.

AUTHOR CORRESPONDENCE: Marie Fournier, PhD, Sanofi, 1 Avenue Pierre Brossolette, 91380 Chilly-Mazarin, France. Tel.: 331604977 77; E-mail: Marie.Fournier@sanofi.com.

\section{DISCLOSURES}

This study was funded by Sanofi and Regeneron Pharmaceuticals. Kiss and Gal are employees of Evidera, which received consulting fees from Sanofi/ Regeneron for conducting this study. Muszbek was employed by Evidera at the time of this study. Kuznik and Chen are current employees of and stockholders in Regeneron Pharmaceuticals. Fournier is an employee of and stockholder in Sanofi. Proudfoot is a former employee of and current stockholder in Sanofi and current employee and stockholder in ViiV Healthcare/GlaxoSmithKline. Michaud has received grant funding from Pfizer and the Rheumatology Research Foundation. The sponsors were involved in the study design, collection, analysis, and interpretation of data as well as data checking of information provided in the manuscript. The authors had unrestricted access to study data, were responsible for all content and editorial decisions, and received no honoraria related to the development of this publication.

\section{ACKNOWLEDGMENTS}

The authors thank Thi-Minh-Thao Huynh for input in the design and analysis of the network meta-analysis comparing licensed DMARDs for the treatment of rheumatoid arthritis; Susan Boklage for input in the cost-utility analysis study design; and Yuxin Li for assistance in programming the cost-utility analytical model and data collection. Medical writing assistance and editorial support under the direction of the authors were respectively provided by Gauri Saal, MA Economics, and Sinead Stewart of Prime (Knutsford, UK), funded by Sanofi/Regeneron according to Good Publication Practice guidelines.

\section{REFERENCES}

1. Arnett FC, Edworthy SM, Bloch DA, et al. The American Rheumatism Association 1987 revised criteria for the classification of rheumatoid arthritis. Arthritis Rheum. 1988;31(3):315-24.

2. Monti S, Montecucco C, Bugatti S, Caporali R. Rheumatoid arthritis treatment: the earlier the better to prevent joint damage. RMD Open. 2015;1(Suppl 1):e000057.

3. Singh JA, Saag KG, Bridges SL Jr, et al. 2015 American College of Rheumatology guideline for the treatment of rheumatoid arthritis. Arthritis Care Res (Hoboken). 2016;68(1):1-25.

4. Smolen JS, Landewé R, Bijlsma J, et al. EULAR recommendations for the management of rheumatoid arthritis with synthetic and biological disease-modifying antirheumatic drugs: 2016 update. Ann Rheum Dis. 2017;76(6):960-77.
5. Baser O, Ganguli A, Roy S, Xie L, Cifaldi M. Impact of switching from an initial tumor necrosis factor inhibitor on health care resource utilization and costs among patients with rheumatoid arthritis. Clin Ther. 2015;37(7):1454-65.

6. Bonafede MM, Curtis JR, McMorrow D, Mahajan P, Chen CI. Treatment effectiveness and treatment patterns among rheumatoid arthritis patients after switching from a tumor necrosis factor inhibitor to another medication. Clinicoecon Outcomes Res. 2016;8:707-15.

7. Chastek B, Becker LK, Chen CI, Mahajan P, Curtis JR. Outcomes of tumor necrosis factor inhibitor cycling versus switching to a disease-modifying anti-rheumatic drug with a new mechanism of action among patients with rheumatoid arthritis. J Med Econ. 2017;20(5):464-73.

8. Rotar Z, Hočevar A, Rebolj Kodre A, et al; Slovenian Rheumatologists. Retention of the second-line biologic disease-modifying antirheumatic drugs in patients with rheumatoid arthritis failing one tumor necrosis factor alpha inhibitor: data from the BioRx.si registry. Clin Rheumatol. 2015;34(10):1787-93.

9. Markenson JA, Gibofsky A, Palmer WR, et al. Persistence with anti-tumor necrosis factor therapies in patients with rheumatoid arthritis: observations from the RADIUS registry. J Rheumatol. 2011;38(7):1273-81.

10. Harrold LR, Reed GW, Magner R, et al. Comparative effectiveness and safety of rituximab versus subsequent anti-tumor necrosis factor therapy in patients with rheumatoid arthritis with prior exposure to anti-tumor necrosis factor therapies in the United States Corrona registry. Arthritis Res Ther. 2015;17:256

11. Kim HL, Lee MY, Park SY, et al. Comparative effectiveness of cycling of tumor necrosis factor- $\alpha$ (TNF- $\alpha$ ) inhibitors versus switching to non-TNF biologics in rheumatoid arthritis patients with inadequate response to TNF- $\alpha$ inhibitor using a Bayesian approach. Arch Pharm Res. 2014;37(5):662-70.

12. Soliman MM, Hyrich KL, Lunt M, et al; British Society for Rheumatology Biologics Register. Rituximab or a second anti-tumor necrosis factor therapy for rheumatoid arthritis patients who have failed their first antitumor necrosis factor therapy? Comparative analysis from the British Society for Rheumatology Biologics Register. Arthritis Care Res (Hoboken). 2012;64(8):1108-15.

13. Curtis JR, Chastek B, Becker L, et al. Further evaluation of a claimsbased algorithm to determine the effectiveness of biologics for rheumatoid arthritis using commercial claims data. Arthritis Res Ther. 2013;15(2):404.

14. Zhou ZY, Griffith J, Du EX, Chin D, Betts KA, Ganguli A. Economic burden of switching to a non-tumor necrosis factor inhibitor versus a tumor necrosis factor inhibitor biologic therapy among patients with rheumatoid arthritis. Adv Ther. 2016;33(5):807-23.

15. Harnett J, Wiederkehr D, Gerber R, Gruben D, Koenig A, Bourret J. Real-world evaluation of TNF-inhibitor utilization in rheumatoid arthritis. J Med Econ. 2016;19(2):91-102.

16. Gottenberg JE, Brocq O, Perdriger A, et al. Non-TNF-targeted biologic vs a second anti-TNF drug to treat rheumatoid arthritis in patients with insufficient response to a first anti-TNF drug: a randomized clinical trial. JAMA. 2016;316(11):1172-80.

17. Wei W, Knapp K, Wang L, et al. Treatment persistence and clinical outcomes of tumor necrosis factor inhibitor cycling or switching to a new mechanism of action therapy: real-world observational study of rheumatoid arthritis patients in the United States with prior tumor necrosis factor inhibitor therapy. Adv Ther. 2017;34(8):1936-52.

18. Finckh A, Ciurea A, Brulhart L, et al. Which subgroup of patients with rheumatoid arthritis benefits from switching to rituximab versus alternative anti-tumour necrosis factor (TNF) agents after previous failure of an antiTNF agent? Ann Rheum Dis. 2010;69(2):387-93.

19. Kamal KM, Madhavan SS, Hornsby JA, Miller LA, Kavookjian J, Scott V. Use of tumor necrosis factor inhibitors in rheumatoid arthritis: a national survey of practicing United States rheumatologists. Joint Bone Spine. 2006;73(6):718-24. 


\section{Cost-Effectiveness of Sarilumab Added to Methotrexate in the Treatment of Adult Patients with Moderately to Severely Active Rheumatoid Arthritis Who Have Inadequate Response or Intolerance to Tumor Necrosis Factor Inhibitors}

20. Reynolds A, Koenig AS, Bananis E, Singh A. When is switching warranted among biologic therapies in rheumatoid arthritis? Expert Rev Pharmacoecon Outcomes Res. 2012;12(3):319-33.

21. Favalli EG, Biggioggero M, Marchesoni A, Meroni PL. Survival on treatment with second-line biologic therapy: a cohort study comparing cycling and swap strategies. Rheumatology (Oxford). 2014;53(9):1664-68.

22. Bergman MJ, Elkin EP, Ogale S, Kamath T, Hamburger MI. Response to biologic disease-modifying anti-rheumatic drugs after discontinuation of anti-tumor necrosis factor alpha agents for rheumatoid arthritis. Rheumatol Ther. 2014;1(1):21-30

23. Chatzidionysiou K, van Vollenhoven RF. Rituximab versus anti-TNF in patients who previously failed one TNF inhibitor in an observational cohort. Scand J Rheumatol. 2013;42(3):190-95.

24. Institute for Clinical and Economic Review. Targeted immune modulators for rheumatoid arthritis: effectiveness \& value. Evidence report. April 7, 2017. Available at: https://icer-review.org/wp-content/uploads/2016/08/NE_CEPAC RA_Evidence_Report_FINAL_040717.pdf. Accessed September 26, 2019.

25. Fisher A, Bassett K, Wright JM, Brookhart MA, Freeman HJ, Dormuth CR Prescriber preference for a particular tumour necrosis factor antagonist drug and treatment discontinuation: population-based cohort. BMJ Open. 2014:4(9):e005532

26. Genovese MC, Fleischmann R, Kivitz AJ, et al. Sarilumab plus methotrexate in patients with active rheumatoid arthritis and inadequate response to methotrexate: results of a phase III study. Arthritis Rheumatol. 2015;67(6):1424-37

27. Burmester GR, Rubbert-Roth A, Cantagrel A, et al. Efficacy and safety of subcutaneous tocilizumab versus intravenous tocilizumab in combination with traditional DMARDs in patients with RA at week 97 (SUMMACTA) Ann Rheum Dis. 2016:75(1):68-74.

28. Strand V, Kosinski M, Chen CI, et al. Sarilumab plus methotrexate improves patient-reported outcomes in patients with active rheumatoid arthritis and inadequate responses to methotrexate: results of a phase III trial. Arthritis Res Ther. 2016;18:198.

29. Fleischmann R, van Adelsberg J, Lin Y, et al. Sarilumab and nonbiologic disease-modifying antirheumatic drugs in patients with active rheumatoid arthritis and inadequate response or intolerance to tumor necrosis factor inhibitors. Arthritis Rheumatol. 2017:69(2):277-90.

30. Abdel-Nasser AM, Rasker JJ, Valkenburg HA. Epidemiological and clinical aspects relating to the variability of rheumatoid arthritis. Semin Arthritis Rheum. 1997;27(2):123-40.

31. Davis S, Stevenson M, Tappenden P, Wailoo A; National Institute for Health and Care Excellence. NICE DSU technical support document 15: cost-effectiveness modelling using patient-level simulation. April 2014. Available at: https://www.ncbi.nlm.nih.gov/books/NBK310370/. Accessed September 26, 2019

32. Caro JJ, Briggs AH, Siebert U, Kuntz KM, Force I-SMGRPT. Modeling good research practices--overview: a report of the ISPOR-SMDM Modeling Good Research Practices Task Force-1. Med Decis Making. 2012;32(5):667-77.

33. Aletaha D, Neogi T, Silman AJ, et al. 2010 rheumatoid arthritis classification criteria: an American College of Rheumatology/European League Against Rheumatism collaborative initiative. Arthritis Rheum. 2010;62(9):2569-81.

34. Choy E, Freemantle N, Proudfoot C, et al. Indirect treatment comparison of the efficacy and safety of sarilumab monotherapy in rheumatoid arthritis patients with inadequate response to conventional disease-modifying antirheumatic drugs. Adv Ther. 2019;36(4):817-27.

35. Smolen JS, Kay J, Doyle MK, et al. Golimumab in patients with active rheumatoid arthritis after treatment with tumour necrosis factor alpha inhibitors (GO-AFTER study): a multicentre, randomised, double-blind, placebo-controlled, phase III trial. Lancet. 2009;374(9685):210-21.

36. Bruce B, Fries JF. The Health Assessment Questionnaire (HAQ). Clin Exp Rheumatol. 2005;23(5 Suppl 39):S14-18.
37. Stevenson M, Archer R, Tosh J, et al. Adalimumab, etanercept, infliximab, certolizumab pegol, golimumab, tocilizumab and abatacept for the treatment of rheumatoid arthritis not previously treated with disease-modifying antirheumatic drugs and after the failure of conventional disease-modifying antirheumatic drugs only: systematic review and economic evaluation. Health Technol Assess. 2016;20(35):1-610.

38. National Institute for Health and Care Excellence. Adalimumab, etanercept and infliximab for the treatment of rheumatoid arthritis. Technology appraisal guidance TA130. October 22, 2007. Available at: https://www.nice. org.uk/guidance/tal30. Accessed September 26, 2019.

39. Diamantopoulos A, Benucci M, Capri S, et al. Economic evaluation of tocilizumab combination in the treatment of moderate-to-severe rheumatoid arthritis in Italy. J Med Econ. 2012;15(3):576-85

40. Wailoo AJ, Bansback N, Brennan A, Michaud K, Nixon RM, Wolfe F. Biologic drugs for rheumatoid arthritis in the Medicare program: a costeffectiveness analysis. Arthritis Rheum. 2008;58(4):939-46.

41. Bansback NJ, Brennan A, Ghatnekar O. Cost effectiveness of adalimum$\mathrm{ab}$ in the treatment of patients with moderate to severe rheumatoid arthritis in Sweden. Ann Rheum Dis. 2005;64(7):995-1002.

42. Boggs R, Sengupta N, Ashraf T. UT3 estimating health utility from a physical function assessment in rheumatoid arthritis (RA) patients treated with adalimumab (D2E7). Value Health. 2002;5(6):452-53.

43. Michaud K, Vera-Llonch M, Oster G. Mortality risk by functional status and health-related quality of life in patients with rheumatoid arthritis. J Rheumatol. 2012;39(1):54-59.

44. Arias E. United States life tables, 2011. Natl Vital Stat Rep. 2015;64(11):1-63.

45. Wailoo A, Brennan A, Bansback N, Nixon R, Wolfe F, Michaud K. Modeling the cost effectiveness of etanercept, adalimumab and anakinra compared to infliximab in the treatment of patients with rheumatoid arthritis in the Medicare program. October 12, 2006. Available at: http:// www.cms.gov/Research-Statistics-Data-and-Systems/Statistics-Trends-andReports/Reports/Research-Reports-Items/CMS1202288.html. Accessed September 26, 2019.

46. Bureau of Labor Statistics. Consumer Price Index. 2018. Available at: http://stats.bls.gov/cpi/. Accessed September 26, 2019.

47. Marra CA, Marion SA, Guh DP, et al. Not all "quality-adjusted life years" are equal. J Clin Epidemiol. 2007;60(6):616-24.

48. Sanders GD, Neumann PJ, Basu A, et al. Recommendations for conduct, methodological practices, and reporting of cost-effectiveness analyses: second panel on cost-effectiveness in health and medicine. JAMA. 2016;316(10):1093-103

49. Fonarow GC, Keech AC, Pedersen TR, et al. Cost-effectiveness of evolocumab therapy for reducing cardiovascular events in patients with atherosclerotic cardiovascular disease. JAMA Cardiol. 2017;2(10):1069-78.

50. Anderson JL, Heidenreich PA, Barnett PG, et al. ACC/AHA statement on cost/value methodology in clinical practice guidelines and performance measures: a report of the American College of Cardiology/American Heart Association Task Force on Performance Measures and Task Force on Practice Guidelines. J Am Coll Cardiol. 2014;63(21):2304-22.

51. Neumann PJ, Sanders GD. Cost-effectiveness analysis 2.0. N Engl J Med. 2017;376(3):203-05.

52. Briggs A, Sculpher M, Claxton K. Decision Modelling for Health Economic Evaluation. New York: Oxford University Press; 2000.

53. Huynh T, Proudfoot C, Chen C, et al. Network meta-analysis of the efficacy and safety of sarilumab monotherapy and combination therapy in rheumatoid arthritis patients with intolerance or inadequate response to diseasemodifying antirheumatic drugs. Paper presented at: Academy of Managed Care Pharmacy Nexus; October 16-29, 2017; Dallas, TX.

54. Vashisht P, O'Dell J. Not all TNF inhibitors in rheumatoid arthritis are created equal: important clinical differences. Expert Opin Biol Ther. 2017;17(8):989-99. 


\section{Cost-Effectiveness of Sarilumab Added to Methotrexate in the Treatment of Adult Patients with Moderately to Severely Active Rheumatoid Arthritis Who Have Inadequate Response or Intolerance to Tumor Necrosis Factor Inhibitors}

55. American College of Rheumatology. Position statement: step therapy. February 2019. Available at: https://www.rheumatology.org/Portals/O/Files/ Step\%20Therapy.pdf. Accessed September 26, 2019.

56. Whittington MD, McQueen RB, Ollendorf DA, et al. Assessing the value of sarilumab monotherapy for adults with moderately to severely active rheumatoid arthritis: a cost-effectiveness analysis. J Manag Care Spec Pharm. 2019;25(1):80-87. Available at: https://www.jmcp.org/doi/10.18553/ jmcp.2019.25.1.080.

57. Fournier M, Chen CI, Kuznik A, Proudfoot C, Mallya UG, Michaud K. Sarilumab monotherapy compared with adalimumab monotherapy for the treatment of moderately to severely active rheumatoid arthritis: an analysis of incremental cost per effectively treated patient. Clinicoecon Outcomes Res. 2019;11:117-28
58. National Institute for Health and Care Excellence. Adalimumab, etanercept, infliximab, rituximab and abatacept for the treatment of rheumatoid arthritis after the failure of a TNF inhibitor. Technology appraisal guidance TA195. August 25, 2010. Available at: https://www.nice.org.uk/guidance/ ta195. Accessed September 26, 2019

59. Curtis JR, Jain A, Askling J, et al. A comparison of patient characteristics and outcomes in selected European and U.S. rheumatoid arthritis registries. Semin Arthritis Rheum. 2010;40(1):2-14.e1.

60. Ramsey SD, Willke RJ, Glick H, et al. Cost-effectiveness analysis alongside clinical trials II-An ISPOR Good Research Practices Task Force report. Value Health. 2015;18(2):161-72. 
Cost-Effectiveness of Sarilumab Added to Methotrexate in the Treatment of Adult Patients with Moderately to Severely Active Rheumatoid Arthritis Who Have Inadequate Response or Intolerance to Tumor Necrosis Factor Inhibitors

\section{APPENDIX A Model Flow}

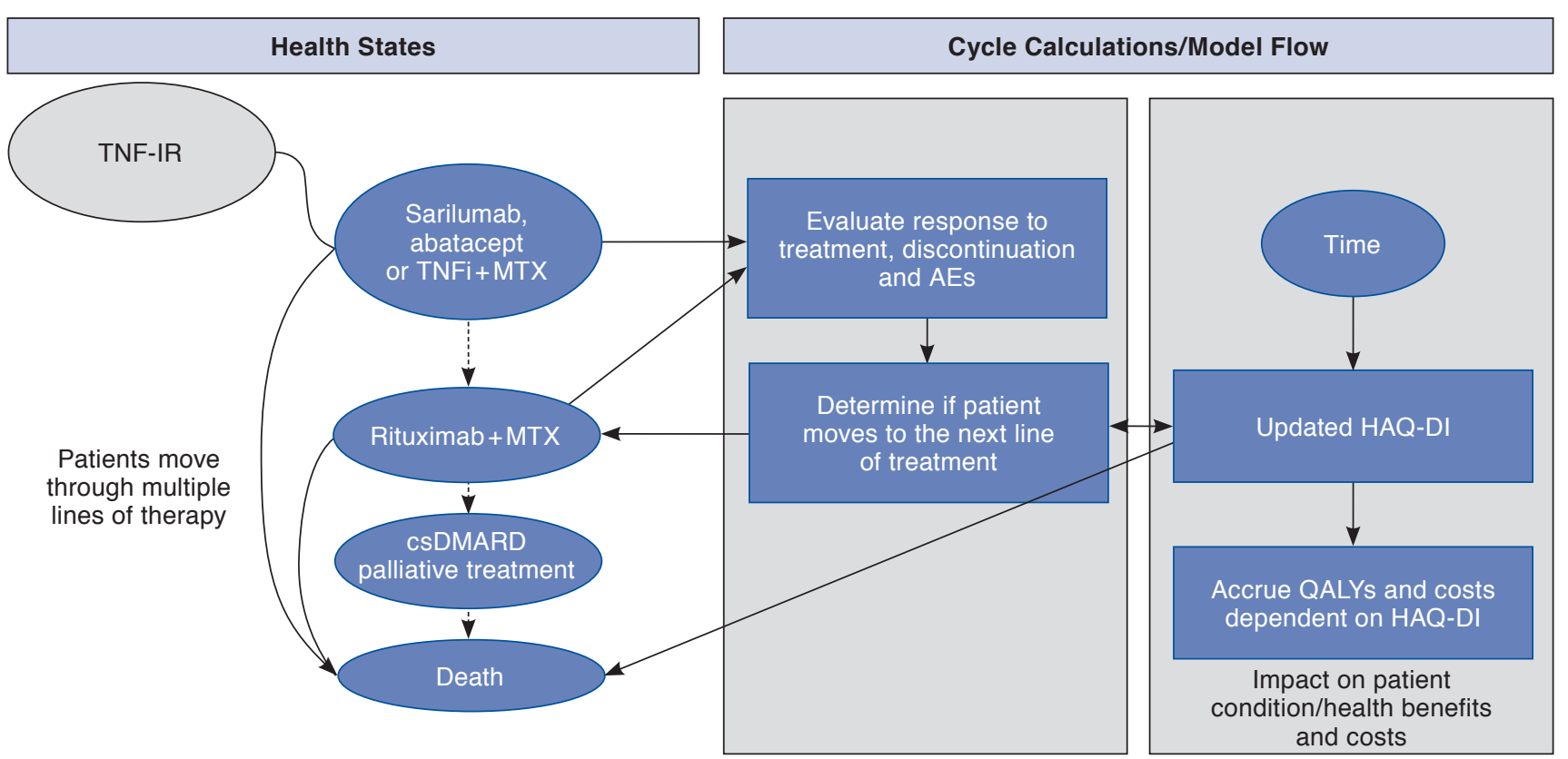

Note: Comparators include abatacept $125 \mathrm{mg}$ SC q1w + methotrexate; a bundle of TNFi agents including adalimumab $40 \mathrm{mg}$ SC q2w + methotrexate, certolizumab $200 \mathrm{mg}$ SC q2w + methotrexate, etanercept $50 \mathrm{mg}$ SC q1w+ methotrexate, and golimumab $50 \mathrm{mg}$ SC q4w+ methotrexate.

$A E s=$ adverse events; $c s D M A R D=$ conventional synthetic disease-modifying antirheumatic drug; HAQ-DI = Health Assessment Questionnaire Disability Index;

MTX=methotrexate; $q 1 w=$ every week; $q 2 w=$ every 2 weeks; $q 4 w=$ every 4 weeks; $Q A L Y$ s = quality-adjusted life-years; SC=subcutaneous; TNF-IR=inadequate response or intolerance to TNFi; TNFi= tumor necrosis factor-alpha inhibitor. 
Cost-Effectiveness of Sarilumab Added to Methotrexate in the Treatment of Adult Patients with Moderately to Severely Active Rheumatoid Arthritis Who Have Inadequate Response or Intolerance to Tumor Necrosis Factor Inhibitors

APPENDIX B Cost-Effectiveness Efficiency Frontier

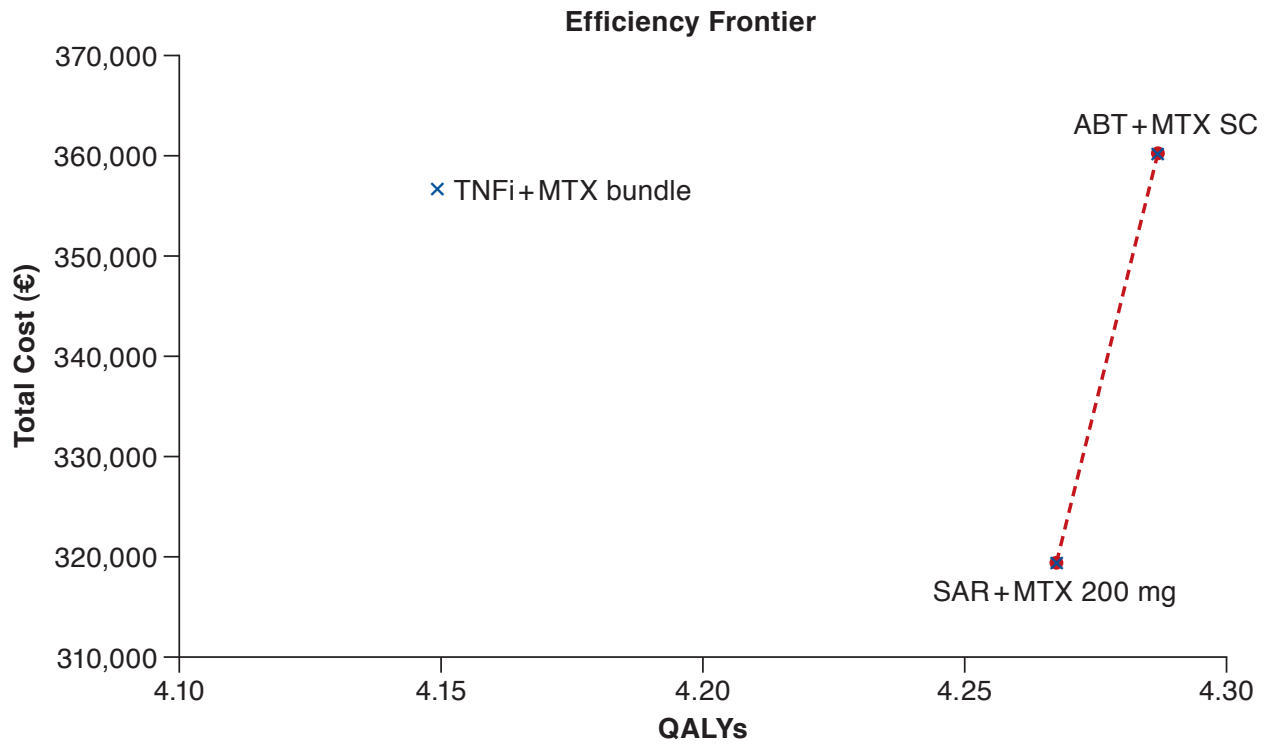

$A B T=$ abatacept; $M T X=$ methotrexate; QALYs = quality-adjusted life-years; SAR=sarilumab; SC=subcutaneous; TNFi=tumor necrosis factor-alpha inhibitor. 